\title{
TRANSFONTANELLAR \\ ULTRASOUND: ASSESSMENT IN NICU
}

F. BENNAOUI1,2, M.LAGRINE 1, 2, N. EL IDRISSI SLITINE1,2, D.BASRAOUI 3, F.M.R MAOULAININE

1,2

1. Neonatal Intensive Care Department, Mohammed VI University Hospital

2. Research Team for Childhood, Health and Development, Marrakech School of Medicine, Cadi Ayyad

University, Marrakech.

3. Department of Medical Radiology CHU Mohammed VI, Marrakech Morocco

\section{INTRODUCTION}

- Transfontanellar ultrasonography is an available, non-invasive, non-irradiating and repetitive imaging technique. It constitutes the initial assessment of cerebral exploration in the newborn and plays a fundamental role in detecting different brain lesions and monitoring their evolution.

-The interest of our study is to devalue the place of the ETF in the neonatology department and to detect the different brain lesions according to the neonatal diseas.

\section{Materials \& Methods :}

- Retrospective study, conducted at Neonatal Intensive Care Department, Mohamed VI University Hospital of Marrakech.

-Duration: from 01/09/2010 to 01/09/2017.

-The population of our study: all newborns receiving an ETF during the study period.

\section{$\underline{\text { Results }}$}

-We collected 447 newborns, including 269 male and 178 female.

-The term newborns constituted 247 cases and the premature 200cas (Graph I).

-The ETF was performed as part of the systematic assessment of preterm infants and in the newborn term in perinatal asphyxia in $55 \%$ of cases, followed by neonatal seizures in $35 \%$ of cases.

-In case of prematurity the ETF had revealed: intraventricular hemorrhage $(\mathbf{4 5 \%})$ followed by periventricular leukomalacia $(8.5 \%)$. (Graph II).

-In the newborn, the ETF had shown in $25.1 \%$ of cases a hyperechogenicity of the thalamus followed by ventricular dilatation in $20.24 \%$ of cases. (Graph III)

\section{DISCUSSION}

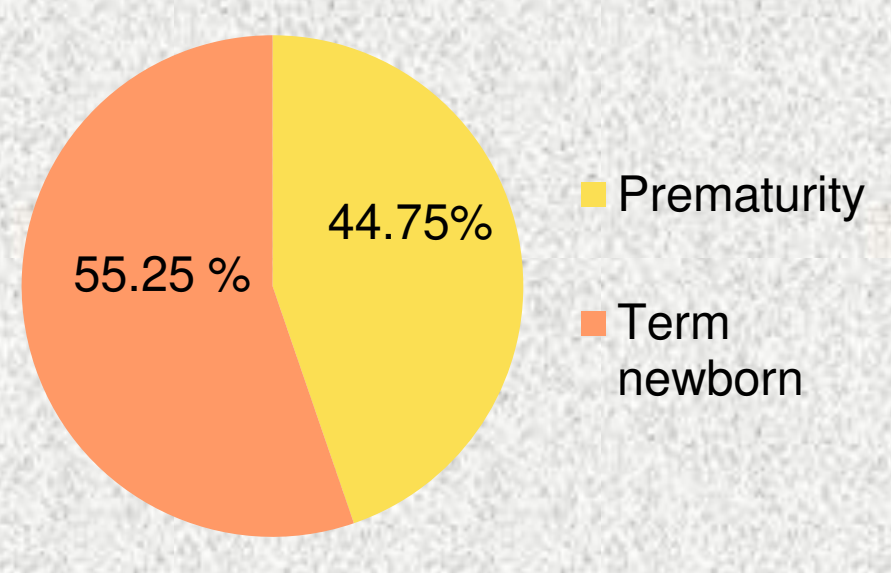

Chart I : Répartition by term

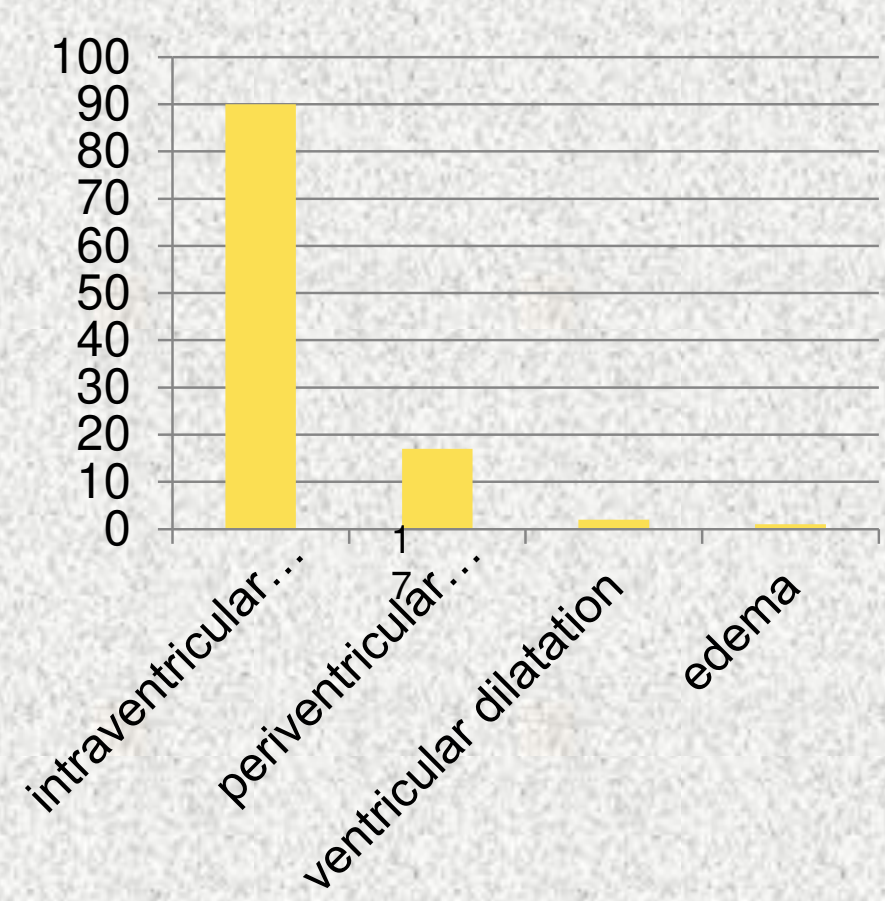

Chart II : ETF abnormalities in term newborn
- ETF is a technique particularly suited to term newborns and premature infants.

-The most common brain lesions in premature infants are intraventricular hemorrhages, which are the most common ETF pathology of prematurity. Its frequency and intensity depend on the term and hemodynamic stability of the newborn. This is explained by fluctuations in cerebral blood flow, related to neonatal hemodynamic conditions and adaptation to assisted ventilation, accompanied by hypertensive blows that cause bleeding.

- Periventricular leucomalacia, thus, this essentially ventricular and periventricular location of the lesions lends itself very well to the transfontanellar pathway; which makes the ultrasound a very sensitive examination for the detection of these pathologies, and therefore particularly adapted to the surveillance of these children.

- In the term neonate, anoxo-ischemic encephalopathy remains the predominant pathology, its topography is rather cortical, the ETF in this context; remains little contributive in the detection of these lesions and complement by MRI is often necessary. In addition to the haemorrhagic and ischemic pathology in the newborn.

- The ETF is able to detect cerebral localized infections including ventriculites, abscesses and empyemas and track their evolution, as well as a number of brain malformations

\section{CONCLUSION}

-The ETF is the simple first-line test to be performed as early as possible, allows diagnosis and follow-up.

-Required for screening for typical lesions of prematurity.

-An MRI is indicated if necessary. 